\title{
Estimation of Human Finger Tapping Forces Based on a Fingerpad-Stiffness Model
}

\author{
Keisuke Shima, Yasuhiro Tamura, Toshio Tsuji, Akihiko Kandori, Masaru Yokoe, and Saburo Sakoda
}

\begin{abstract}
This paper proposes a method of estimating fingertip forces in finger tapping movements based on human fingerpad characteristics. Since the human fingerpad exhibits elasticity, the proposed technique recreates the relationship between the fingertip force and the displacement generated between the thumb and index fingerpads as a fingerpadstiffness model. Then, using this model, the force between the two fingertips (the fingertip force) can be estimated from the measured fingerpad deformation only. As the method does not require any sensors to be attached to the finger contact surface to measure fingertip force, it can be used to evaluate the tendency of force in natural and unconstrained finger tapping movements conducted by the subject.

In the experiments conducted, fingertip forces and the displacement of the two fingerpads generated when the subjects pinched and pushed a force sensor with the thumb and index finger were measured to approximate the relationships between fingerpad force and deformation. The results indicated that human fingerpad characteristics can be expressed using a fingerpad-stiffness function (including an exponential function), and that fingerpad forces can be estimated using the proposed model. Furthermore, comparison between a Parkinson's disease (PD) patient and a healthy subject confirmed differences in the finger tapping forces for each. This implies the possibility of assessing motor function in PD patients using the finger tapping force evaluation method proposed in this paper.
\end{abstract}

\section{INTRODUCTION}

The Unified Parkinson's Disease Rating Scale (UPDRS) [1] part III (motor score), which includes hand open-close movement, pronosupination and finger tapping movement, is a standard outcome scale for disability and impairment in Parkinson's disease. In particular, finger tapping movements have been widely applied in clinical environments to evaluate motor function impairment caused by cerebral infarction or neurological disorders. However, it is difficult even for highly trained doctors to quantitatively evaluate motor ability and determine slight changes in symptoms.

The quantification of finger tapping movements has already been extensively investigated through techniques such as evaluating tapping rhythms, velocities and amplitudes using electrocardiographic apparatus, a musical instrument digital interface (MIDI) keyboard [2], an infrared camera [3], an acceleration sensor [4] and a magnetic sensor [5],

This study was supported in part by a Grant-in-Aid for JSPS Fellows (19 - 9510) and Grant-in-Aid for Scientific Research (B) (21360118) from the Japan Society for the Promotion of Science.

K. Shima, Y. Tamura, and T. Tsuji are with Graduate School of Engineering, Hiroshima University, Higashi-hiroshima, 739-8527 Japan shimalbsys.hiroshima-u.ac.jp

A. Kandori is with Fundamental Research Laboratory, Hitachi Ltd., Kokubunji, 185-8601 Japan

M. Yokoe and S. Sakoda are with Graduate School of Medicine, Osaka University, Suita, 565-0871 Japan
[6]. However, these studies measured and discussed only parameters of voluntary/involuntary movement such as tapping rhythms and velocities. For investigation to evaluate human movement, it is necessary to consider that voluntary human action is realized by motor commands from the brain to the muscles, resulting in muscle activation with force output. Measuring the electrical activity of the cerebral nervous system and muscle force information will enable evaluation of the internal condition of people with motor impairments and elucidation of finger movements for the assessment of motor function.

Some discussion on methods of measuring finger tapping forces directly using strain gages has already been made [7], [8]. For the measurement of force, it should be noted that attaching a sensor to the finger contact surface (i.e., the fingerpad) causes an adverse effect on natural finger tapping movement. To overcome this problem, Okuno et al. [9] proposed a force estimation method that uses an acceleration sensor attached to the fingernails of the thumb and index finger to identify the relationship between fingertip acceleration and force in finger tapping movements. However, the acceleration sensor is affected not only by finger acceleration but also by hand acceleration, making it potentially difficult to evaluate forces in slow finger movements with no acceleration.

In this paper, we propose a method to estimate the force generated between two fingerpads (the fingerpad force) without attaching a sensor to the finger contact surface. Using this method based on a fingerpad-stiffness model that can express the relationships between fingerpad force and deformation, the fingerpad force can be estimated by measuring fingerpad deformation only. The magnetic sensor [5], [6] with coils attached to the fingernails that is used to measure fingertip distances in this study enables evaluation of fingerpad forces in natural and unconstrained finger tapping movements. The forces estimated with this method can therefore be used as a new index for assessing neurological disorders such as Parkinson's disease (PD) in subjects.

\section{ESTIMATION METHOD FOR FINGER TAPPING FORCES}

\section{A. Fingerpad-stiffness model}

First, let us consider a finger pressed on a fixed object as an example. In this case, fingertip forces are generated on the fingerpad (known as fingerpad forces) with some degree of strain deformation in the pad. Since the human fingerpad exhibits elasticity, it is clear that fingerpad characteristics can be at least partially expressed by a stiffness function. For ease of explanation, the degree of strain deformation in 


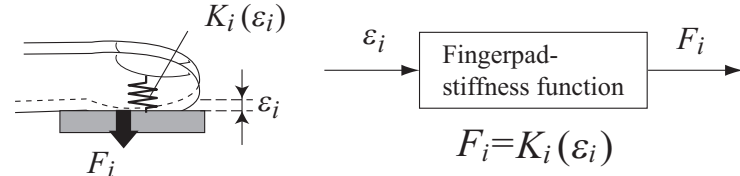

(a) Fingerpad-stiffness model

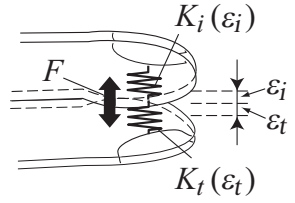

(b) Force between two fingers

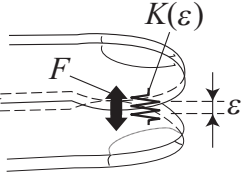

(c) Relation between force and displacement
Fig. 1. Overview of fingertip stiffness characteristics

a fingerpad and variations in stress are identified as fingerpad deformation $\varepsilon_{i}$ (i.e., to fingertip displacement) by onedimensional mapping from a lateral view of the fingertips and the force perpendicular to the plane of the object $F_{i}$. Based on this concept, the relationships between fingerpad force and deformation can be assumed as the stiffness function, where the input is fingertip displacement and the output is force (Fig. 1(a)). In the case of fingers (i.e., the thumb and index finger) squeezing together, each fingerpad is deformed according to the force generated between the two fingertips. The amplitude of force between the fingertips $F$ can therefore be expressed as follows (see Fig. 1(b)):

$$
F=K_{t}\left(\varepsilon_{t}\right) \varepsilon_{t}=K_{i}\left(\varepsilon_{i}\right) \varepsilon_{i},
$$

where $\varepsilon_{t}$ and $\varepsilon_{i} \geq 0$ are the fingerpad deformation of the thumb and index finger, respectively, and $K_{t}\left(\varepsilon_{t}\right)$ and $K_{i}\left(\varepsilon_{i}\right)$ describe the fingerpad-stiffness function of each finger. In this case, the forces generated are balanced between each fingerpad, and the stiffness function of the two fingerpads in a state of contact can be seen as a series stiffness model. The fingerpad-stiffness function for both fingers can thus be expressed as

$$
\begin{aligned}
\frac{1}{K\left(\varepsilon_{t}, \varepsilon_{i}\right)} & =\frac{1}{K_{t}\left(\varepsilon_{t}\right)}+\frac{1}{K_{i}\left(\varepsilon_{i}\right)} \\
& =\frac{\varepsilon_{t}}{F}+\frac{\varepsilon_{i}}{F} \\
& =\frac{\varepsilon_{t}+\varepsilon_{i}}{F} .
\end{aligned}
$$

This paper therefore approximates the relationships between the fingerpad deformation of both fingers $\varepsilon=\varepsilon_{t}+\varepsilon_{i}$ and the fingerpad force as follows (see Fig. 1(c)):

$$
F=K(\varepsilon) \varepsilon .
$$

Using this method, the fingerpad-stiffness function $K(\varepsilon)$ is identified from measured fingerpad deformation $\varepsilon$ and force $F$ using the least-square method for the estimation of finger tapping force.

\section{B. Estimation of finger tapping forces}

Figure 2 shows an outline of the proposed force estimation method. First, the fingertip distance $d(t)$ at the instance of

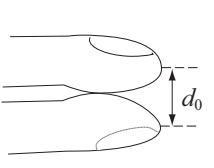

(a) $d(t)=d_{0}$

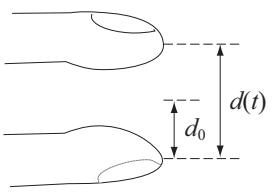

(b) $d(t)>d_{0}$

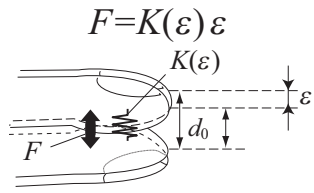

(c) $d(t)<d_{0}$
Fig. 2. Proposed method for force estimation in finger tapping

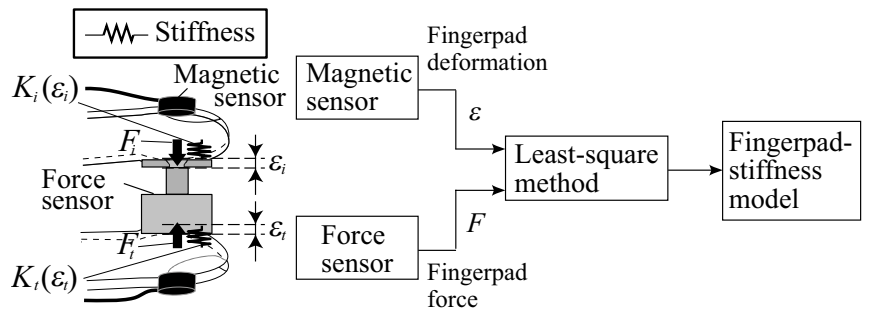

Fig. 3. Outline of the experiment for force estimation

finger contact during finger tapping movements is defined as $d_{0}$ (Fig. 2(a)). The system judges that the fingers are in an open position when $d(t)>d_{0}$, and the fingertip forces are then set as $F=0$ (Fig. 2(b)). When $d(t)$ is less than $d_{0}$ (indicating the period of finger contact), the fingerpads are deformed by fingerpad forces (Fig. 2(c)), and this fingerpad deformation can be expressed as

$$
\varepsilon=d_{0}-d(t) .
$$

Using $\varepsilon$ and the fingerpad-stiffness function identified, the fingertip forces are calculated using Eq. 5.

\section{EXPERIMENTS}

To verify the validity of the proposed method, fingertapping force estimation experiments were conducted with six healthy subjects (A-F; five males and one female; average age: $23.0 \pm 0.6$ ) and a PD patient (G; male; age: 75; UPDRS part III finger tapping score: 2).

\section{A. Experimental equipment}

Figure 3 shows an outline of the experiments conducted in this study. In these tests, a magnetic sensor (Hitachi Ltd.), a three-axis force sensor (Nitta Corporation) and a multitelemeter system (Web-5000; Nihon Kohden Corporation) were used. The magnetic sensor can output voltages corresponding to changes in the distance between the two coilbearing fingertips (the fingertip distance), and the output voltages of the sensor are converted to fingertip distances based on nonlinear calibration (the details are described in [6]). The force measured by the force sensor is filtered using a second-order Butterworth low-pass filter (cutoff frequency: $1 \mathrm{~Hz}$ ). Electromyogram (EMG) signals are measured and amplified using the multi-telemeter system, and the integrated EMG (IEMG) is then computed at time intervals of $0.1 \mathrm{~s}$. The muscle contraction level (MCL) computed from IEMG signals using the maximal voluntary contraction (MVC) values for each subject is utilized to evaluate the tendencies of the subject's force. The EMG electrodes are 


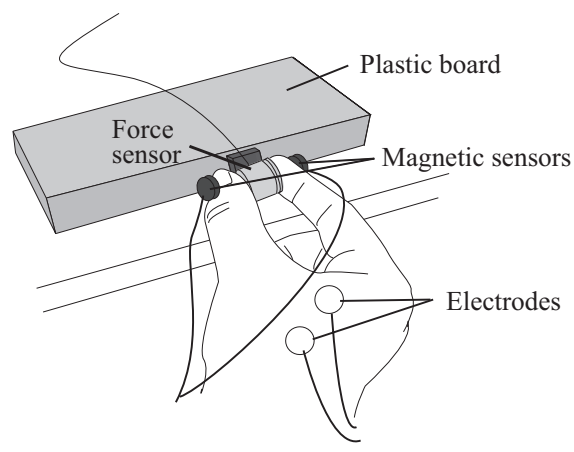

Fig. 4. Experimental apparatus for identification of fingerpad-stiffness function

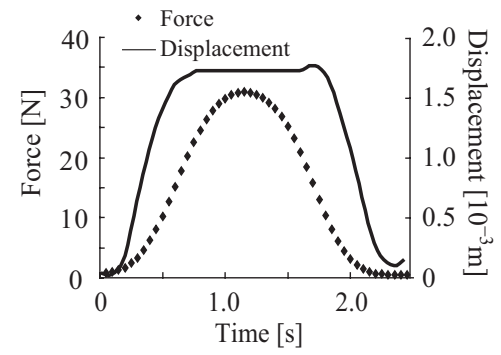

Fig. 5. Example of measured force and displacement

set on the first dorsal interosseous muscle (FDI), the extensor carpi ulnaris (ECU) and the flexor carpi ulnaris (FCU). The sampling frequency of the magnetic sensor and force sensor were $100 \mathrm{~Hz}$, and EMG signals were measured with 2000 $\mathrm{Hz}$ sampling.

\section{B. Identification of the fingerpad-stiffness function}

1) Methods: The fingerpad-stiffness function was identified, and was used to estimate the fingertip forces to demonstrate the effectiveness of the proposed method. Figure 4 shows the experimental setup for identification. The subjects were first asked to pinch the force sensor under nondeformation conditions of the fingerpads. The force sensor was supported with the opposite hand of the subject if necessary, and the finger contact points on the force sensor were kept in the same position for all trials using support apparatus (Fig. 4). The subject then pressed slowly on the sensor with a force of about $30 \mathrm{~N}$ while keeping the fingerpads parallel to each other. One set of force increase/decrease between $0 \mathrm{~N}$ and $30 \mathrm{~N}$ was defined as a single trial, and the total number of trials was five. After the measurement, the fingertip forces and displacement (fingerpad deformation) were used to identify the fingerpad-stiffness function.

Furthermore, to check the accuracy of force estimation, the estimated forces were compared with the measured values for consecutive pressing of the force sensor. The subjects were asked to press the sensor with three levels of force (about 5, 15 and $30 \mathrm{~N}$ ), and five consecutive taps were conducted in each case (i.e., the total number of taps was 15). To investigate the influence of fingerpad elasticity on estimation accuracy, the experiments were conducted for the two cases of increasing force (subjects pressed the sensor with an

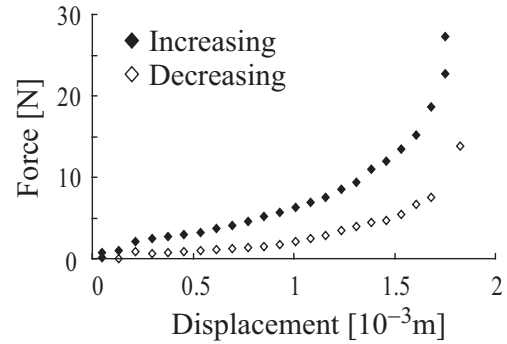

Fig. 6. Displacement-force characteristics

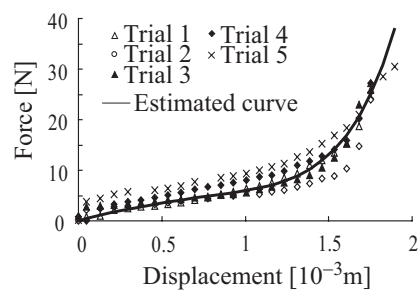

(a) Increasing force

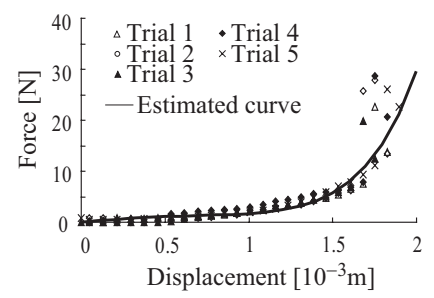

(b) Decreasing force
Fig. 7. Measured and estimated displacement-force characteristics

approximate force from $5 \mathrm{~N}$ to $30 \mathrm{~N}$ ) and decreasing force (from $30 \mathrm{~N}$ to $5 \mathrm{~N}$ ). A total of five trials were conducted.

2) Results and discussion: Examples of the measured results for fingerpad deformation and force are shown in Figs. 5 and 6. From Fig. 6, it can be seen that the relationship between fingerpad deformation and force forms a hysteresis curve. Figures 7 (a) and (b) show the measurement data for all trials in the cases of increasing and decreasing force, respectively. The data for each figure change exponentially for fingerpad deformation through all trials. The fingerpadstiffness function is therefore modeled in this paper as follows:

$$
K(\varepsilon)=\sum_{n=0}^{N} \alpha_{n} e^{n \varepsilon},
$$

where $\alpha_{n}$ is a constant estimated from fingerpad force and deformation using the linear least-square method for each subject. The approximated curves of the deformation-force characteristics computed using Eq. 7 with $N=2$ are shown in Figs. 7 (a) and (b). From these figures, it is confirmed that the approximated curves express the relationships between fingerpad deformation and force. The mean square error between the estimated and measured forces for all subjects is $0.28 \pm 0.10 \mathrm{~N}$.

The results of comparison between the estimated and measured forces are shown in Fig. 8, and those for the correlation coefficients with all subjects are shown in Fig. 9. Figure 8 indicates that the estimated forces change in a similar way to the measured forces. In the case of decreasing force, however, the estimated values vary widely when the force is large. It should be noted that estimation accuracy is high for forces under $10 \mathrm{~N}$, with a mean square estimation error of $0.16 \pm 0.10 \mathrm{~N}$. Furthermore, high coefficients of correlation between the estimated and measured forces are observed for all subjects. Since the average values of the 


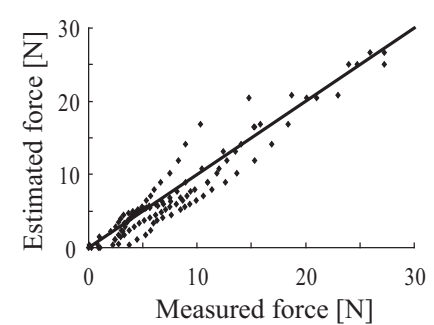

(a) Increasing force

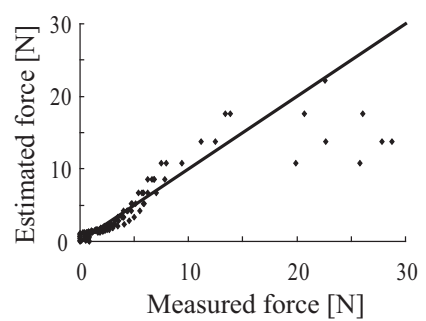

(b) Decreasing force
Fig. 8. Comparison between the estimated and measured forces

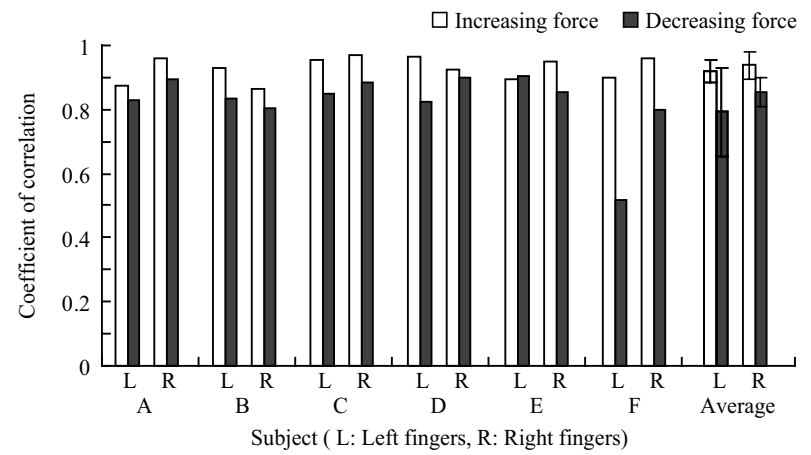

Fig. 9. Coefficients of correlation between the estimated and measured forces

correlation coefficient for all subjects is high (for increasing force: $0.93 \pm 0.04$; for decreasing force: $0.82 \pm 0.10$ ), we concluded that the tendency of fingertip forces could be estimated from fingerpad deformation using the fingerpadstiffness function in Eq. 7.

For the case of consecutive pressing on the force sensor, examples of the measured results is shown in Fig. 10, and the correlation coefficients between the estimated and measured forces for all subjects are also shown in Fig. 11. The average values of the correlation coefficients for all subjects in the case of increasing and decreasing forces are $0.85 \pm 0.05$ and $0.82 \pm 0.06$, respectively. These results indicate that the proposed model can estimate fingerpad force and deformation characteristics with high accuracy.

\section{Finger-tapping force estimation experiments}

1) Methods: To identify the effectiveness of the proposed method for real-time estimation, we conducted experiments to compare the forces estimated and the IEMG signals measured from natural and unconstrained finger tapping movements. The subjects were asked to conduct finger tapping with three degrees of force (weak, moderate and strong) at time intervals of $10 \mathrm{~s}$. The fingerpad forces were then evaluated from the fingerpad deformation generated during finger contact using the fingerpad-stiffness function identified in III-B. Here, the fingerpad-stiffness functions of increasing force for each subject, which showed the highest estimation accuracy in the identification experiments, were used to estimate fingerpad forces. One trial was conducted. Furthermore, the estimated forces were compared between a normal subject and a PD patient.

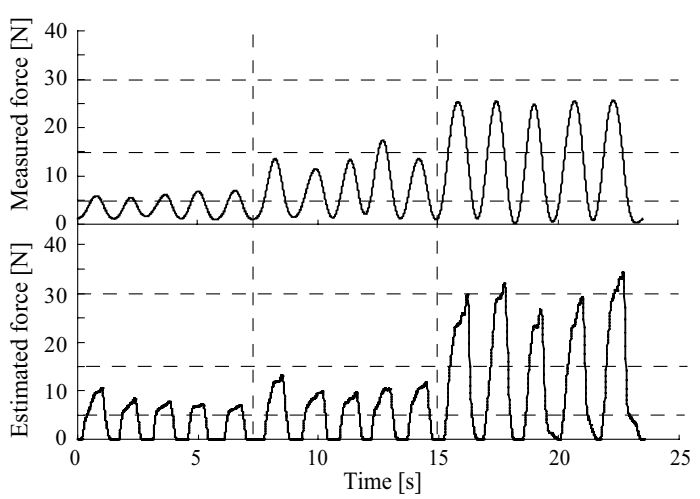

(a) Increasing force

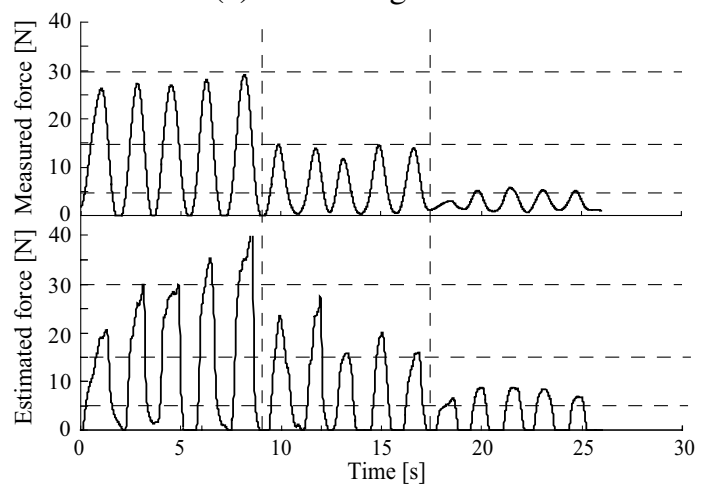

(b) Decreasing force

Fig. 10. The estimated and measured forces in consecutive presses

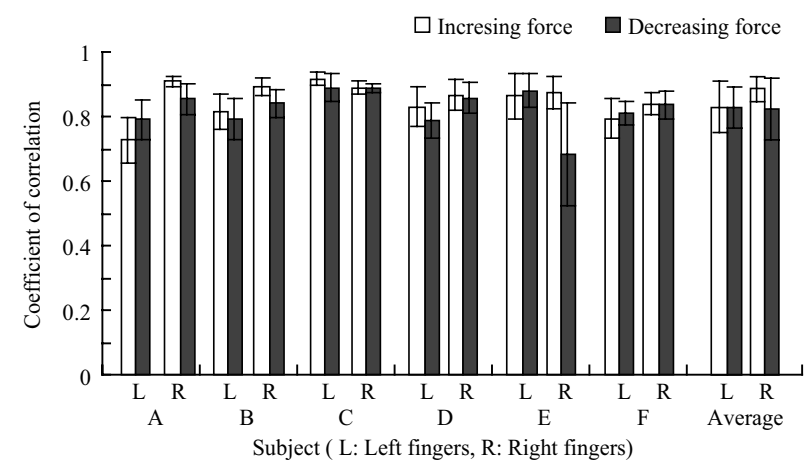

Fig. 11. Coefficients of correlation between the estimated and measured forces in consecutive presses

2) Results and discussion: Figure 12 shows examples of the experimental results in the case of increasing force, and plots the distance between the two fingertips $d(t)$ measured by a magnetic sensor, fingertip displacement $\varepsilon$ (i.e., fingerpad deformation), estimated force $F(\varepsilon)$ and IEMG signals of FDI. Here, the subjects were asked to change the degree of force at each instant (10 and $20 \mathrm{~s}$, shown by the dotted lines in Fig. 12). From this figure, it can be seen that the fingerpad deformation becomes larger in line with increasing IEMG signals, which is similar to the tendency of the estimated forces. In each single tap, the maximum values of estimated force and the average values of IEMG signals during finger contact were computed and compared to investigate the accuracy of force estimation. The coefficient of correlation between them for all finger taps is $0.67 \pm$ 


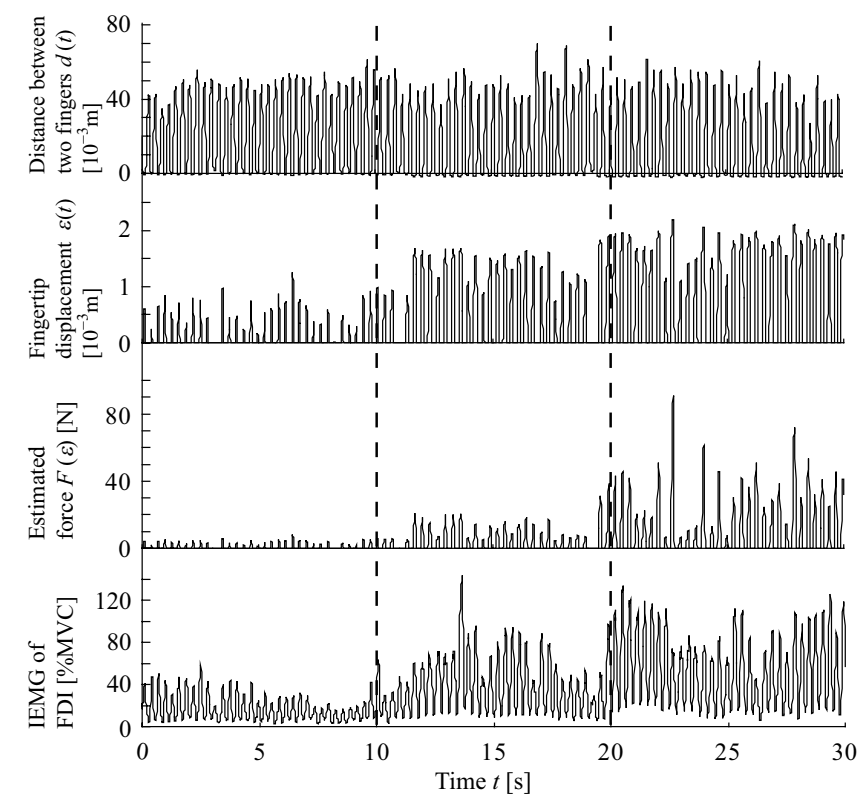

Fig. 12. An example of estimated finger tapping forces

0.08 , indicating that the estimated force correlates roughly with the IEMG signals. These results lead us to conclude that the proposed finger-tapping force estimation method can be used to evaluate the tendency of force generated in finger tapping movements through the measurement of fingerpad deformation without attaching a sensor to the finger contact surface. Furthermore, Fig 13 shows an example of the estimated forces with a normal subject and a PD patient. It shows that the movements and forces of the PD patient vary widely compared with those of the normal subject. These results indicate that the proposed method can be used to evaluate the motor function of PD patients.

\section{CONCLUSION}

This paper proposes a fingerpad-stiffness model that can evaluate the forces generated in fingerpads during finger tapping movements. By measuring fingerpad deformation when one finger comes into contact with another using a non-contact sensor (such as a magnetic sensor) for the finger contact surface, the fingerpad force can be estimated in real time. In the experiments conducted, the relationships between fingerpad force and deformation were modeled using a fingerpad-stiffness function (including an exponential function) with six subjects. The correlation coefficients between the estimated and measured forces were $0.93 \pm$ 0.04 (in the case of increasing force) and $0.82 \pm 0.10$ (in the case of decreasing force). Furthermore, fingerpad forces measured during finger tapping movements were compared with measured IEMG signals, and the average coefficient of correlation between them was $0.67 \pm 0.08$. These results indicate that fingerpad forces can be evaluated by measuring fingerpad deformation only.

In future work, measurement experiments need to be conducted with normal elderly subjects and patients with

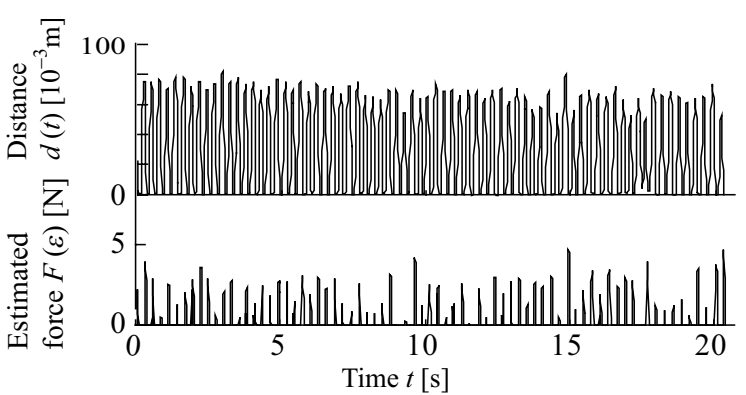

(a) A normal subject

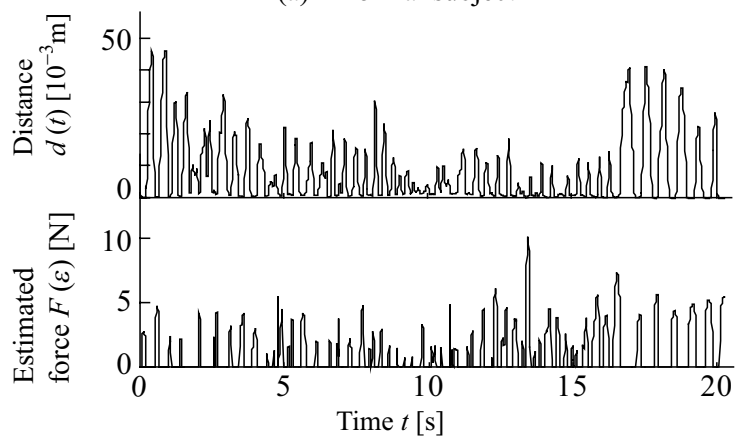

(b) A Parkinson's disease patient

Fig. 13. Measured results of figer-tapping movements and estimated force

motor function impairment such as PD in order to investigate the effects of aging and disease. We also plan to apply and discuss more complex functions (such as those for viscosity) in the fingerpad-stiffness model to improve the approximation accuracy of human fingerpad characteristics.

\section{REFERENCES}

[1] S. Fahn, R. L. Elton, Members of The UPDRS Development Committee: Unified Parkinson's Disease Rating Scale, In: S. Fahn, C. D. Marsden, D. B. Calne, M. Goldstein, "Recent Developments in Parkinson's Disease," Macmillan Health Care Information, Vol. 2, pp. 153-163, 1987.

[2] J. Konczak, H. Ackermann, I. Hertrich, S. Spieker, J. Dichgans, "Control of repetitive lip and finger movements in Parkinson's disease," Movement Disorders, vol. 12, no. 5, pp. 665-676, 1997.

[3] R. Agostino, A. Curra, M. Giovannelli, N. Modugno, M. Manfredi, A. Berardelli, "Impairment of individual finger movements in Parkinson's disease," Movement Disorders, vol. 18, no. 5, pp. 560-565, 2003.

[4] M. Yokoe, R. Okuno, T. Hamasaki, Y. Kurachi, K. Akazawa and S. Sakoda, "Opening velocity, a novel parameter, for finger tapping test in patients with Parkinson's disease," Parkinsonism and Related Disorders, 2008 (in press)

[5] A. Kandori, M. Yokoe, S. Sakoda et al.' Quantitative magnetic detection of finger movements in parients with Parkinson's disease, Neuroscience Reseach, Vol. 49, No. 2, pp. 253-260, 2004

[6] K. Shima, T. Tsuji, E. Kan, A. Kandori, M. Yokoe, and S. Sakoda "Measurement and Evaluation of Finger Tapping Movements Using Magnetic Sensors," Proceedings of the 30th Annual International Conference of the IEEE Engineering in Medicine and Biology Society, pp. 5628-5631, 2008.

[7] P. N. Loseby, J. P. Piek, N. C. Barret' The influence of speed and force on bimanual finger tapping pattarns, Human Movement Science, Vol. 20, No. 4-5, pp. 531-547, 2001

[8] N. Inui, H. Hotta' Asymmetric control of force and symmetric control of timing in bimanual finger tapping, Human Movement Science, Vol. 21, No. 2, pp. 131-146, 2002

[9] R. Okuno, M. Yokoe, K. Akazawa, K. Abe, S. Sakoda, "Finger taps acceleration measurement system for quantitative diagnosis of Parkinson's disease," Proceedings of the 2006 IEEE International Conference of the Engineering in Medicine and Biology Society, pp. 6623-6626, 2006. 